J. Nonlinear Var. Anal. 2 (2018), No. 2, pp. 229-232

Available online at http://jnva.biemdas.com

https://doi.org/10.23952/jnva.2.2018.2.09

\title{
DISINTEGRATION OF YOUNG MEASURES AND CONDITIONAL PROBABILITIES
}

\author{
TORU MARUYAMA \\ Department of Economics, Keio University, Tokyo, Japan
}

\begin{abstract}
Existence of a version of conditional probabilies is proved by means of a general disintegration theorem of Young measures due to M. Valadier.

Keywords. Conditional probability; Disintegration; Young measure.
\end{abstract}

2010 Mathematics Subject Classification. 28A50, 28A51, 60B10.

\section{INTRODUCTION}

In this brief note, the relationship between the concept of disintegrations of Young measures and that of regular conditional probabilities is clarified. Although the similarity between these two has been noticed in the long history of the theory of Young measures, some vagueness seems to have been left. Our target is to prove the existence of regular conditional probabilities through the disintegrability theorem. For a general theory of Young measures, the readers are referred to Valadier [5].

\section{PRELIMINARIES}

Let $(\Omega, \mathscr{E}, \mu)$ be a finite measure space and $X$ a Hausdorff topological space endowed with the Borel $\sigma$-field $\mathscr{B}(X)$. The mapping $\pi_{\Omega}: \Omega \times X \rightarrow \Omega$ (resp. $\pi_{X}: \Omega \times X \rightarrow X$ ) denotes the projection of $\Omega \times X$ into $\Omega$ (resp. $X$ ).

A (positive) finite measure $\gamma$ on $(\Omega \times X, \mathscr{E} \otimes \mathscr{B}(X))$ is called a Young measure if it satisfies

$$
\gamma \circ \pi_{\Omega}^{-1}=\mu .
$$

The set of all Young measures on $(\Omega \times X, \mathscr{E} \otimes \mathscr{B}(X))$ is denoted by $\mathfrak{Y}(\Omega, \mu ; X)$.

A family $\left\{v_{\omega} \mid \omega \in \Omega\right\}$ of measures on $(X, \mathscr{B}(X))$ is called a measurable family if the mapping

$$
\omega \mapsto v_{\omega}(B)
$$

is measurable for any $B \in \mathscr{B}(X)$. Given a measurable family $\left\{v_{\omega} \mid \omega \in \Omega\right\}$ of finite measures on $(X, \mathscr{B}(X))$, the function

$$
\omega \mapsto \int_{X} \chi_{A}(\omega, x) d v_{\omega}
$$

is measurable for any $A \in \mathscr{E} \otimes \mathscr{B}(X) . \chi_{A}$ is the characteristic function of $A$. A set function $\gamma$ defined by

$$
\gamma(A)=\int_{\Omega}\left\{\int_{X} \chi_{A}(\omega, x) d v_{\omega}\right\} d \mu, \quad A \in \mathscr{E} \otimes \mathscr{B}(X)
$$

E-mail address: maruyama@econ.keio.ac.jp.

Received December 25, 2017; Accepted February 16, 2018.

(C)2018 Journal of Nonlinear and Variational Analysis 
is a measure on $(\Omega \times X, \mathscr{E} \otimes \mathscr{B}(X))$. In particular if every $v_{\omega}(\omega \in \Omega)$ is a probability measure, then $\gamma$ defined by (2.2) is a Young measure since (2.1) is satisfied.

Conversely, is it possible to represent any Young measure on $\Omega \times X$ in the form of (2.2) for some measurable family $\left\{v_{\omega} \mid \omega \in \Omega\right\}$ of probability measures? The most general result concerning this problem is given by Valadier [4].

If a Young measure $\gamma$ is representable in the form (2.2), its right-hand side is called the disintegration of $\gamma$. In this case, we symbolically express $\gamma$ as

$$
\gamma=\int_{\Omega} \delta_{\omega} \otimes v_{\omega} d \mu
$$

\section{VALADIER's DisinTEGRABILITY THEOREM}

Valadier's quite general result on the disintegrability of Young measures is stated below.

Let $\mathscr{E}$ be a $\sigma$-field on $\Omega$. The completion of $\mathscr{E}$ by a finite (positive) measure $\mu$ is denoted by $\mathscr{E} \mu$. A function $f: \Omega \rightarrow \mathbb{R}$ is said to be $\mathscr{E}_{\mu}$-measurable if it is measurable with respect to $\mathscr{E}_{\mu}$.

Theorem 3.1 (Valadier's Disintegration Theorem (Valadier [4] )). Let $(\Omega, \mathscr{E}, \mu)$ be a finite (positive) measure space and $X$ a Hausdorff topological space. Suppose that $\gamma$ is a Young measure on $(\Omega \times X, \mathscr{E} \otimes$ $\mathscr{B}(X))$ such that $\gamma \circ \pi_{X}^{-1}$ is a Radon measure on $X$. Then there exists a $\mathscr{E}_{\mu}$-measurable family $\left\{v_{\omega} \mid \omega \in \Omega\right\}$ of Radon probability measures on $X$ which satisfies

$$
\gamma(A)=\int_{\Omega}\left\{\int_{X} \chi_{A}(\omega, x) d v_{\omega}\right\} d \mu \quad \text { for } \quad A \in \mathscr{E}_{\mu} \otimes \mathscr{B}(X) .
$$

Remark 3.1. Valadier's proof makes use of the theory of liftings due to A. and C. Ionescu-Tulcea. In case that $X$ is a metrizable space enjoying some additional properties, the proof becomes a little bit easy. (cf. Valadier [5] pp.178-182.)

Remark 3.2. If $X$ is a Souslin space, any finite Borel measure on it is automatically Radon.

\section{Conditional Probabilities}

Let $(\Omega, \mathscr{E}, P)$ be a probability space and $\mathfrak{F}$ a sub- $\sigma$-field of $\mathscr{E}$. As anyone knows, the existence of the conditional probability $P(A \mid \mathfrak{F})(\omega)(A \in \mathscr{E})$ is assured by means of Radon-Nikodým theorem. $P(A \mid \mathfrak{F})(\omega)$ is said to be regular if (i) the mapping $\omega \mapsto P(A \mid \mathfrak{F})(\omega)$ is $\mathfrak{F}$-measurable for any $A \in \mathscr{E}$ and (ii) $P(\cdot \mid \mathfrak{F})(\omega)$ is a probability measure on $(\Omega, \mathscr{E})$ for a.e. $\omega$. A similarity between the concept of regular conditional probabilities and that of measurable families is quite clear.

We now proceed to the existence problem of regular conditional probabilities. The following theorem is basically obtained by Hoffmann-Jørgensen [2] and Chatterji [1]. However we now give an alternative proof based upon Valadier's theorem. (See also Schwartz [3].)

Theorem 4.1. Let $(\Omega, \mathscr{E})$ be a measurable space and $X$ a Hausdorff topological space with a Radon probability measure $\theta$. A function $p: X \rightarrow \Omega$ is assumed to be $(\mathscr{B}(X), \mathscr{E})$-measurable. A measure $\mu$ on $(\Omega, \mathscr{E})$ is defined by $\mu=\theta \circ p^{-1}$. Then there exists a $\mathscr{E}_{\mu}$-measurable family $\left\{v_{\omega} \mid \omega \in \Omega\right\}$ of Radon probabilities on $X$ which satisfies

$$
\int_{\Omega} v_{\omega}\left(B \cap p^{-1}(A)\right) d \mu=\theta\left(B \cap p^{-1}(A)\right) \quad \text { for } \quad A \in \mathscr{E}, B \in \mathscr{B}(X) .
$$

(The extension of $\mu$ is also denoted by the same notation.) 
Proof. To start with, we define a measure $\gamma$ on $(\Omega \times X, \mathscr{E} \otimes \mathscr{B}(X))$ by

$$
\gamma(E)=\int_{\Omega}\left\{\int_{X} \chi_{E}(\omega, x) d \theta\right\} d \mu \quad \text { for } \quad E \in \mathscr{E}_{\mu} \otimes \mathscr{B}(X) .
$$

We can easily confirm that $\gamma$ is a Young measure, taking account of the relation

$$
\begin{aligned}
\gamma \circ \pi_{\Omega}^{-1}(A) & =\int_{\Omega}\left\{\int_{X} \chi_{A \times X}(\omega, x) d \theta\right\} d \mu \\
& =\int_{\Omega} \chi_{A}(\omega) d \mu \\
& =\mu(A) \text { for } A \in \mathscr{E} .
\end{aligned}
$$

We also note that

$$
\begin{aligned}
\gamma \circ \pi_{X}^{-1}(B) & =\int_{\Omega}\left\{\int_{X} \chi_{\Omega \times B}(\omega, x) d \theta\right\} d \mu \\
& =\int_{\Omega} \theta(B) d \mu \\
& =\theta(B) \quad \text { for } \quad B \in \mathscr{B}(X) .
\end{aligned}
$$

Hence $\gamma \circ \pi_{X}^{-1}$ is a Radon measure on $X$.

By Valadier's theorem, there exists a $\mathscr{E}_{\mu}$-measurable family $\left\{v_{\omega} \mid \omega \in \Omega\right\}$ of Radon probabilities on $X$ which satisfies

$$
\gamma(E)=\int_{\Omega}\left\{\int_{X} \chi_{E}(\omega, x) d v_{\omega}\right\} d \mu \quad \text { for } \quad E \in \mathscr{E}_{\mu} \otimes \mathscr{B}(X) .
$$

If $E=A \times B \in \mathscr{E} \otimes \mathscr{B}(X)$ in particular, we obtain

$$
\begin{aligned}
\gamma(A \times B) & =\int_{\Omega}\left\{\int_{X} \chi_{A \times B}(\omega, x) d v_{\omega}\right\} d \mu \\
& =\int_{\Omega} \chi_{A}(\omega) v_{\omega}(B) d \mu \\
& =\int_{A} v_{\omega}(B) d \mu .
\end{aligned}
$$

Since $\theta=\gamma \circ \pi_{X}^{-1}$ by (4.1), we find that

$$
\begin{aligned}
\theta\left(B \cap p^{-1}(A)\right) & =\gamma \circ \pi_{X}^{-1}\left(B \cap p^{-1}(A)\right) \\
& =\gamma\left(\Omega \times\left(B \cap p^{-1}(A)\right)\right. \\
& =\int_{\Omega} v_{\omega}\left(B \cap p^{-1}(A)\right) d \mu
\end{aligned}
$$

by (4.2). This completes the proof.

The following result is actually a corollary of Theorem 4.1.

Theorem 4.2. Let $X$ be a Hausdorff topological space endowed with the Borel $\sigma$-field $\mathscr{B}(X)$. $\mu$ is a Radon probability measure on $X$. For any sub- $\sigma$-field $\mathscr{E}$ of $\mathscr{B}(X)$, there exists a $\mathscr{E} \mu$-measurable family $\left\{v_{\omega} \mid \omega \in X\right\}$ which satisfies

$$
\mu(A \cap B)=\int_{A} v_{\omega}(B) d \mu \quad \text { for } \quad A \in \mathscr{E}, B \in \mathscr{B}(X) .
$$

(The extension of $\mu$ is also denoted by the same notation.) 
Proof. Let $\Omega$ be a copy of $X$ (i.e. $\Omega=X$ ) endowed with $\mathscr{E}$. We specify a mapping $p: X \rightarrow \Omega$ as the identity $p(\omega)=\omega$, which is $(\mathscr{B}(X), \mathscr{E})$-measurable.

Since $\theta$ in Theorem 4.1 corresponds to the Radon measure $\mu$ on $(X, \mathscr{B}(X))$ here, $\mu=\theta \circ p^{-1}$ in Theorem 4.1 should be interpreted as $\left.\mu\right|_{\mathscr{E}}$, the restriction of $\mu$ to $\mathscr{E}$.

Applying Theorem 4.1 to this simple setting, there exists a $\mathscr{E}_{\mu}$-measurable family $\left\{v_{\omega} \mid \omega \in \Omega\right\}$ of Radon probability measures on $X$ which satisfies

$$
\begin{array}{r}
\int_{\Omega} \chi_{A}(\omega) v_{\omega}(B) d \mu=\mu\left(B \cap p^{-1}(A)\right)=\mu(A \cap B) \\
\qquad \text { for } A \in \mathscr{E}, B \in \mathscr{B}(X) .
\end{array}
$$

This proves Theorem 4.2 .

\section{REFERENCES}

[1] S. D. Chatterji, Disintegration of measures and lifting, In: D. H. Tucker and H. B. Maynard, (eds.) Vector and Operator Valued Measures and Applications, pp. 69-83, Academic Press, New York/London, 1973.

[2] J. Hoffmann-Jørgensen, Existence of conditional probabilities, Math. Scand. 28 (1971), 257-264.

[3] L. Schwartz, Lectures on Disintegration of Measures, Tata Institute of Fundamental Research, Bombay, 1975.

[4] M. Valadier, Comparaison de troi théorèmes de désintégration, Séminaire d'analyse convexe, 1972.

[5] M. Valadier, Young measures, In: A. Cellina, (ed.) Methods of Nonconvex Analysis, pp. 152-188, Springer-Verlag, Berlin/Heidelberg, 1990. 